\title{
Idade de segregação do lenho juvenil e adulto pela variação da massa específica de açoita-cavalo
}

\author{
Estimating the age of demarcation of young and mature wood in açoita-cavalo
}

\author{
Matheus Lemos de Peres ${ }^{\mathrm{I}}$ Darci Alberto Gatto ${ }^{\mathrm{II}}$ Diego Martins Stangerlin ${ }^{\mathrm{III}}$ Leandro Calegari ${ }^{\mathrm{IV}}$ \\ Rafael Beltrame $^{\mathrm{V}}$ Clóvis Roberto Haselein ${ }^{\mathrm{VI}}$ Elio José Santini ${ }^{\mathrm{VI}}$
}

\section{RESUMO}

O presente trabalho teve como objetivo estimar a idade de segregação do lenho de Luehea divaricata Mart. (açoita-cavalo) por meio da variação radial da massa específica. Para o desenvolvimento do estudo, foram coletadas dez árvores de duas regiões do Estado do Rio Grande do Sul. Foi retirado um disco por árvore, com aproximadamente $2 \mathrm{~cm}$ de espessura a $0,10 \mathrm{~m}$ de altura do tronco. De cada disco, retirou-se, com auxílio de uma serra-fita, uma bagueta central de 2,0cm de largura, bem orientada no sentido radial, incluindo a medula no centro, dividiu-se então a bagueta em duas amostras " $A$ " $e$ " $B$ ". A massa específica foi obtida da relação entre peso seco e volume úmido. O volume úmido foi determinado de acordo com a norma ASTM D 2395 - 93, 1995. A segregação dos lenhos foi encontrada por regressão linear simples dos dados de variação da massa específica no sentido medula-casca. Foi feita uma regressão linear na faixa crescente dos dados e outra na faixa decrescente. A intersecção das retas extrapoladas foi adotada como idade de segregação. Os resultados obtidos mostraram que o lenho de açoita-cavalo segrega em aproximadamente 26 anos para a região da Depressão Central e 23 anos para a região da Encosta Superior do Nordeste. Nos dados de variação de massa específica, foi feita uma análise de variância multifatorial. Constatou-se que há diferença significativa entre lenhos e entre regiões. Por outro lado, as amostras $A$ e $B$ de cada árvore não foram estatisticamente diferentes entre si.

Palavras-chave: densidade; Luehea divaricata; qualidade da madeira.

\begin{abstract}
This study aims to estimate the age of segregation of young and mature wood of Luehea divaricata Mart., using the radial variation of specific gravity. To the development of this study were collected ten trees from two regions of the state of Rio Grande do Sul. Discs with thickness of $2 \mathrm{~cm}$ at $0.1 \mathrm{~m}$ of height from the base of the trunk were used. From each disk, with the aid of a band saw, a central ribbon $2.0 \mathrm{~cm}$ wide, and oriented in the radial direction was taken, including the pith, then splitted into two samples " $A$ " and " $B$ ". The specific gravity was calculated as ratio between dry weight and wet volume. The wet volume was determined according to ASTM D 2395 - 93, 1997. The age of segregation was found by simple linear regression of the data of variation of specific gravity from pith to bark. The intersection of extrapolated lines was adopted as the age of segregation. The results showed that the wood segregates in 26 year for the region of Central Depression and in 23 years for the Northeast Upper Hillside. Was made a multifactorial analysis of variance in the specific gravity data, showing that there are statistical difference between regions and woods. However, there are no statistical difference between samples.
\end{abstract}

Key words: density; Luehea divaricata; wood quality.

\section{INTRODUÇÃO}

As variações da massa específica dependem das mudanças na proporção dos vasos e das

'Programa de Pós-graduação em Ciência e Engenharia de Materiais (PPGCEM), Centro de Desenvolvimento Tecnológico (CDTec), Universidade Federal de Pelotas (UFPel), 96010-000, Pelotas, RS, Brasil. E-mail: matheusldeperes@gmail.com. Autor para correspondência.

"Curso de Engenharia Industrial Madeireira (CCEIM), Centro de Engenharias, UFPel, Pelotas, RS, Brasil.

IIIInstituto de Ciências Agrárias e Ambientais, Universidade Federal de Mato Grosso (UFMT), Sinop, MT, Brasil.

${ }^{\text {IV } U n i d a d e ~ A c a d e ̂ m i c a ~ d e ~ E n g e n h a r i a ~ F l o r e s t a l, ~ U n i v e r s i d a d e ~ F e d e r a l ~ d e ~ C a m p i n a ~ G r a n d e ~(U F C G), ~ P a t o s, ~ P B, ~ B r a s i l . ~}$

vPrograma de Pós-graduação em Engenharia Florestal (PPGEF), Universidade Federal de Santa Maria (UFSM), Santa Maria, RS, Brasil.

vIDepartamento de Ciências Florestais, Centro de Ciências Rurais (CCR), UFSM, Santa Maria, RS, Brasil. 
espessuras das paredes celulares das fibras ou traqueídeos. De acordo com OLIVEIRA \& SILVA(2003), o aumento da espessura da parede celular das fibras ou o aumento na proporção das fibras em relação, por exemplo, à proporção de vasos ocasiona o aumento da massa específica. Do contrário, o aumento na proporção de vasos, com ou sem decréscimo na espessura da parede celular, leva à redução na propriedade citada. Dessa forma, sabe-se que a massa específica depende intrinsecamente de componentes da anatomia da madeira.

A massa específica é de grande importância, pois fornece informações sobre as características da madeira, devido a sua íntima relação com outras propriedades, sendo, assim, um parâmetro utilizado para avaliar as propriedades mecânicas e físicas da madeira nos diversos segmentos da atividade industrial. De acordo com SILVA et al. (2004), a massa específica é uma propriedade resultante de uma combinação dos constituintes anatômicos da madeira.

A influência na diferenciação dos componentes anatômicos da madeira é feita por ações ambientais. Nesse sentido, VALE et al. (2009) afirmam que, ao longo do desenvolvimento da árvore, fatores ambientais e intrínsecos à própria espécie determinam o grau de variação da massa específica no sentido radial e longitudinal, podendo servir como parâmetro, conforme sugerido por KOLLMANN \& CÔTÉ JUNIOR (1968), para separar a madeira em classes de qualidade, em função da posição de retirada da tora na árvore. Essas variações também podem ocorrer, segundo os autores, em razão da anatomia da madeira, pela diferença entre tipos, forma, estrutura e organização das células, diferenciando os lenhos juvenil e adulto.

Portanto, a diferença básica entre o lenho juvenil e o lenho adulto é a variação das dimensões dos componentes anatômicos da madeira. Segundo RAMSAY \& BRIGGS (1986), a madeira juvenil caracteriza-se anatomicamente por um progressivo acréscimo nas dimensões das células e por correspondentes alterações na sua forma, estrutura e disposição em sucessivos anéis de crescimento, além do aumento na massa específica. Tal afirmação possibilita a determinação da segregação dos lenhos em função dos caracteres anatômicos e também em função da variação radial da massa específica.

A determinação da idade aproximada em que ocorre a segregação da madeira juvenil para madeira adulta é de grande importância prática, haja vista a crescente proporção de madeira juvenil no mercado. Segundo BENDTSEN \& SENFT (1986), essa informação permite estimar melhor os valores comuns entre as propriedades de madeira juvenil e adulta e, consequentemente, pode contribuir para uma melhor utilização dos tipos de lenho. Todavia, sabe-se que a madeira adulta apresenta melhores propriedades físicas e mecânicas, portanto, conhecendo-se o ano estimado em que ocorre o início da produção de lenho adulto, pode-se programar o manejo silvicultural, englobando desbaste e desrama, de forma a suprimir a produção de lenho juvenil nos anos antecedentes à segregação do lenho, ou seja, proceder ao desbaste após esta data para que a árvore tenha maior proporção de lenho adulto.

O presente trabalho teve como objetivo estimar a idade aproximada de segregação entre lenhos adulto e juvenil a partir da variação radial da massa específica, além de determinar se há diferença estatística na variação da massa específica entre lenhos, regiões fisiográficas e entre amostras.

\section{MATERIAL E MÉTODOS}

A região da Depressão Central do Estado do Rio Grande do Sul (Figura 1) constitui uma área sem grandes variações altimétricas, em que as maiores cotas se situam ao redor de $200 \mathrm{~m}$, as quais dominam as amplas e alongadas formas de topos convexos ou planos, cujas encostas caem suavemente em direção aos vales, com aprofundamento médio em torno dos 40m (GATTO et al., 2008). A vegetação dessa Região classifica-se em Floresta Estacional Decidual, com temperaturas médias máximas e mínimas de $25^{\circ} \mathrm{C}$ e $14^{\circ} \mathrm{C}$, respectivamente, e precipitação anual média de $1600 \mathrm{~mm}$.

A Encosta Superior do Nordeste está situada entre a Encosta Inferior do Nordeste e os Campos de Cima da Serra. Sua área perfaz um total de $7.683 \mathrm{~km}^{2}$. O relevo é muito montanhoso, de formação geológica basáltica. A Região é recortada profundamente por rios que formam vales, com altitudes de até 800 metros nos limites com o Planalto (GATTO et al., 2008). A vegetação dessa região se classifica em Floresta Ombrófila Mista, com clima caracterizado por temperaturas médias mínimas anuais de $15^{\circ} \mathrm{C}$ e médias máximas de $23^{\circ} \mathrm{C}$, tendo precipitação anual média de $1800 \mathrm{~mm}$.

Para a estimativa da idade de segregação da madeira de açoita-cavalo, foram eleitas cinco árvores de cada Região do Rio Grande do Sul, de forma a serem contempladas as maiores variações de clima do Estado. As árvores foram selecionadas ao acaso, de acordo com a norma COPANT (1971) eASTM D5536-94(1995) e abatidas em floresta nativa (processo de licenciamento pela Secretaria Estadual do Meio Ambiente do Rio Grande do Sul) sem manejo florestal e sem espaçamento fixo. As árvores da espécie citada podem alcançar $30 \mathrm{~m}$ de altura e $100 \mathrm{~cm}$ de DAP 


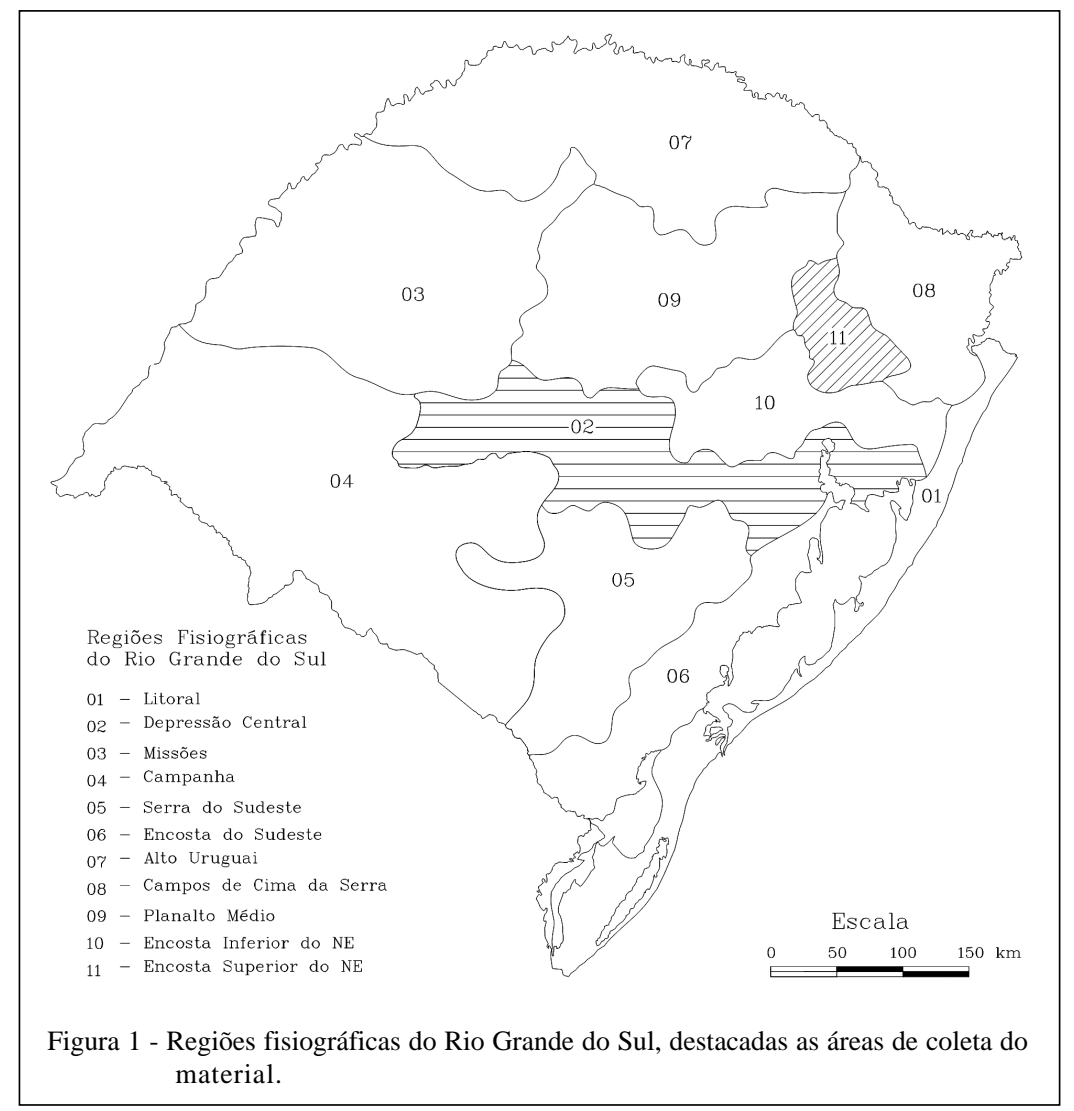

(Diâmetro à Altura do Peito), medido a $130 \mathrm{~cm}$ do solo. A altura do fuste pode alcançar $10 \mathrm{~m}$, tendo copa densa de 4 a $5 m$ de largura.

Na determinação da idade de segregação, usou-se o método de análise da variação da massa específica básica no sentido medula-casca. Para a determinação dos dados de massa específica, usou-se a primeira fatia, coletada a $0,1 \mathrm{~m}$ de altura de cada uma das árvores selecionadas, da qual foi retirada uma bagueta central de $2 \mathrm{~cm}$ de largura bem orientada no sentido radial com a medula no centro, sem orientação cardinal e dividida em duas amostras, sendo denominadas A e B. Após, em laboratório, foram confeccionados corpos-de-prova a cada centímetro (1x2x2cm) no sentido radial (medula-casca) (Figura 2). Os corpos-de-prova foram imersos em água até atingirem massa constante. Destes, foi medido o volume pelo método do deslocamento por imersão em água (ASTM D 2395-93, 1995) e a massa seca, calculando-se a massa específica básica a partir da

seguinte equação: $\mathbf{M E}_{\text {bás }}=\frac{M_{s}}{V u}$, em que: $\mathrm{ME}_{\text {bás }}=$ massa específica básica (g. $\left.\mathrm{cm}^{-3}\right) ; \mathrm{V}_{\mathrm{u}}=$ Volume úmido $\left(\mathrm{cm}^{3}\right) ; \mathrm{M}_{\mathrm{s}}$ $=$ massa seca $(\mathrm{g})$.
Além da determinação da idade de segregação do lenho juvenil e do lenho adulto para a madeira de Luehea divaricata Mart., verificou-se a possível diferença estatística, a 5\% de significância, entre os valores de massa específica para as duas regiões estudadas, entre lenhos e entre as amostras $\mathrm{A}$ e B da mesma bagueta. Para tanto, foram realizadas análises de variância multifatorial com o uso do software estatístico STATGRAPHICS Centurion XV, sendo o fator dependente o conjunto de dados de massa específica e os fatores influentes as duas regiões, os dois lenhos (juvenil e adulto) e as amostras (A e B). No programa, com a utilização da análise de variância multifatorial foram atribuídos os índices 1 e 2 para cada grupo, isto é, para a região da Depressão Central, usouse 1 e, para a Encosta Superior do Nordeste, 2, com a finalidade de diferenciá-los perante o programa. Para a diferenciação entre lenhos e amostras, usou-se o mesmo método.

Para que se determinasse o ponto de segregação do lenho juvenil e do lenho adulto foram ajustadas regressões lineares na nuvem de dados correspondente à variação de massa específica no sentido medula-casca. Essas regressões foram divididas em duas, sendo uma regressão feita na faixa de dados crescente e outra feita na faixa decrescente. 

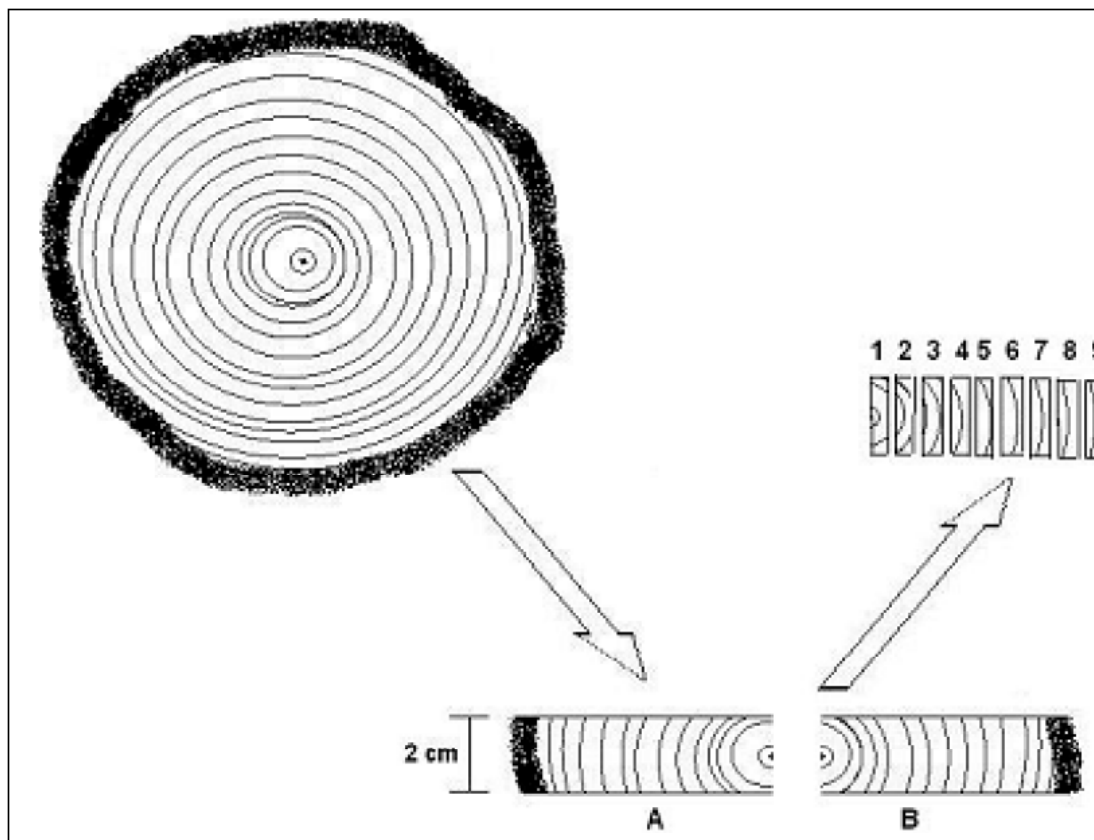

Figura 2 - Método de confecção das amostras para a determinação da variação da massa específica no sentido medula-casca.

Após, fez-se uma extrapolação das retas de regressão, para que se pudesse analisar o ponto em que elas se cruzaram, ou seja, matematicamente, o ponto comum entre as duas, obtido igualando-se as duas equações de reta. O modelo linear de regressão foi escolhido para que pudessem ser cruzadas as duas retas, originando o ponto correspondente ao ano de segregação dos lenhos.

\section{RESULTADOS E DISCUSSÃO}

Na tabela 1, é apresentado o conjunto de médias para massa específica, dividido entre os fatores influentes, ou seja, regiões, lenhos e amostras. Os resultados corroboram os verificados por PANSHIN
\& DE ZEEUW (1980) e SILVA (2004), que afirmam que a massa específica pode variar entre espécies e também entre indivíduos da mesma espécie.

As variações da massa específica no sentido radial, bem como no sentido longitudinal, estão relacionadas com a idade da árvore, amostragem, genótipo e condições ambientais (ALZATE et al., 2005). Dessa forma, concordando com os autores, os resultados obtidos diferem entre Regiões, haja vista a diferença climática entre elas.

As variações estatísticas da massa específica básica a $12 \%$ entre as regiões da Depressão Central e da Encosta Superior do Nordeste estão apresentadas na tabela 2. Pode-se observar que houve diferença estatisticamente significativa, ao nível de 95\% de confiança, entre os lenhos juvenil e adulto,

Tabela 1 - Médias para massa específica com intervalos de 95\% de confiança.

\begin{tabular}{|c|c|c|c|c|}
\hline Nível & Média $\left(\mathrm{g} \mathrm{cm}^{-3}\right)$ & $\mathrm{S}_{\mathrm{xy}}$ & Limite Inferior & Limite Superior \\
\hline \multicolumn{5}{|l|}{ Lenho } \\
\hline Inicial & 0,515556 & 0,002446 & 0,510748 & 0,520365 \\
\hline Tardio & 0,528989 & 0,002297 & 0,524473 & 0,533504 \\
\hline \multicolumn{5}{|l|}{ Região } \\
\hline Depressão Central & 0,515142 & 0,00245 & 0,510327 & 0,519957 \\
\hline Encosta Superior do Nordeste & 0,529404 & 0,002275 & 0,524933 & 0,533874 \\
\hline \multicolumn{5}{|l|}{ Amostra } \\
\hline A & 0,520197 & 0,00252 & 0,515245 & 0,52515 \\
\hline B & 0,524348 & 0,00223 & 0,519964 & 0,528732 \\
\hline
\end{tabular}

Em que: $\mathrm{S}_{\mathrm{xy}}=$ Erro padrão de estimativa. 
Tabela 2 - Análise de variância multifatorial da Massa Específica observada.

\begin{tabular}{lccccc}
\hline Fonte & Soma de Quadrados & Graus de Liberdade & Quadrado Médio & Valor F calculado & P (Probabilidade) \\
\hline Fatores & & & & & \\
Lenho & 0,0189911 & 1 & 0,0189911 & 16,07 & 0,0001 \\
Região & 0,021743 & 1 & 0,021743 & 18,39 & 0,0000 \\
Amostra & 0,0017936 & 1 & 0,0017936 & 1,52 & 0,2187 \\
Resíduos & 0,503559 & 426 & 0,00118206 & & \\
Total (corrigido) & 0,547913 & 429 & & & \\
\hline
\end{tabular}

*Teste de Fisher, com 5\% de significância.

demonstrando que a variação da massa específica é um fator auxiliar na obtenção da idade de segregação dos lenhos.
Na figura 3, tem-se a representação da variação da massa específica básica da madeira de açoita-cavalo no sentido medula-casca para as duas
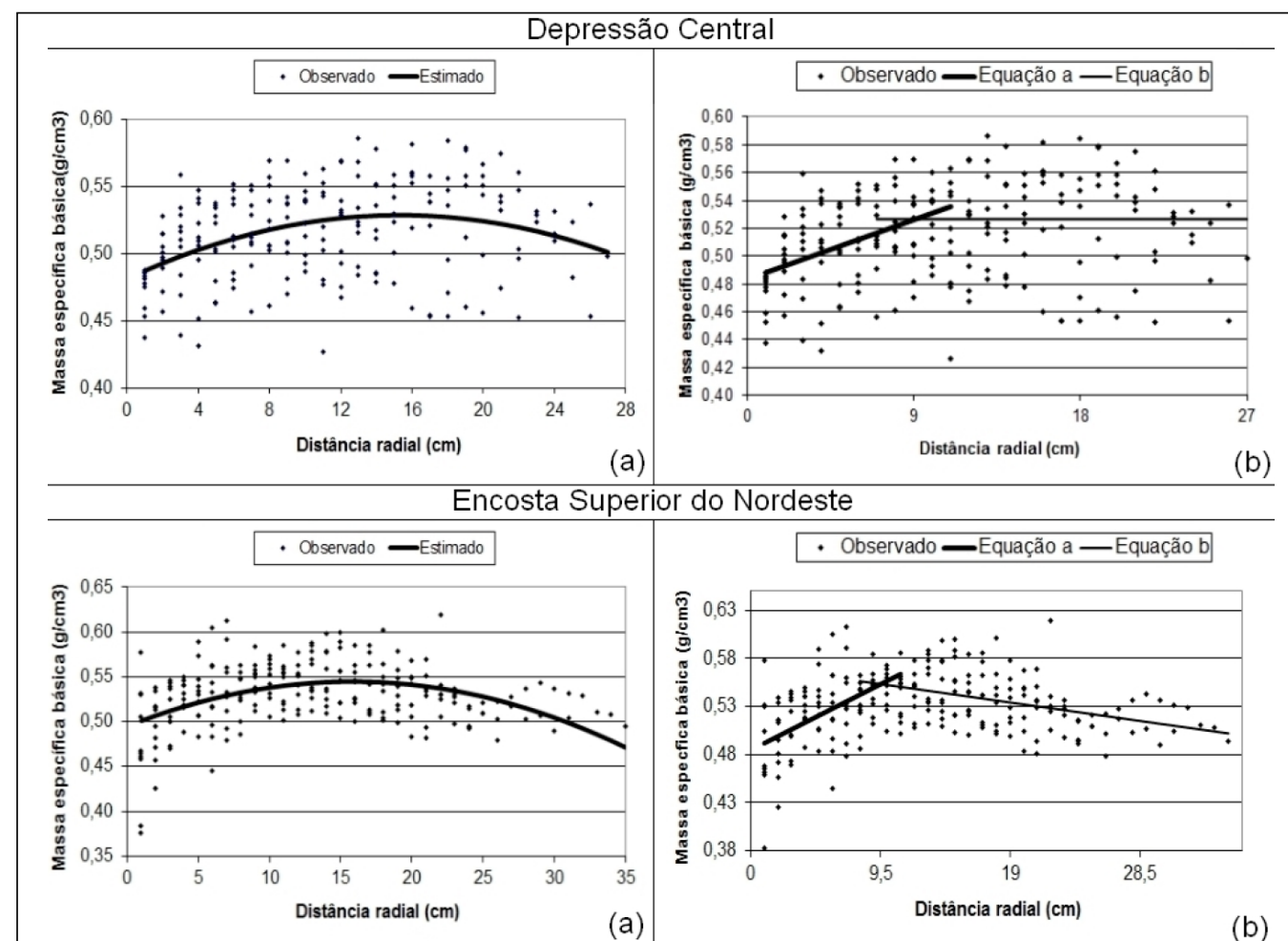

Tota

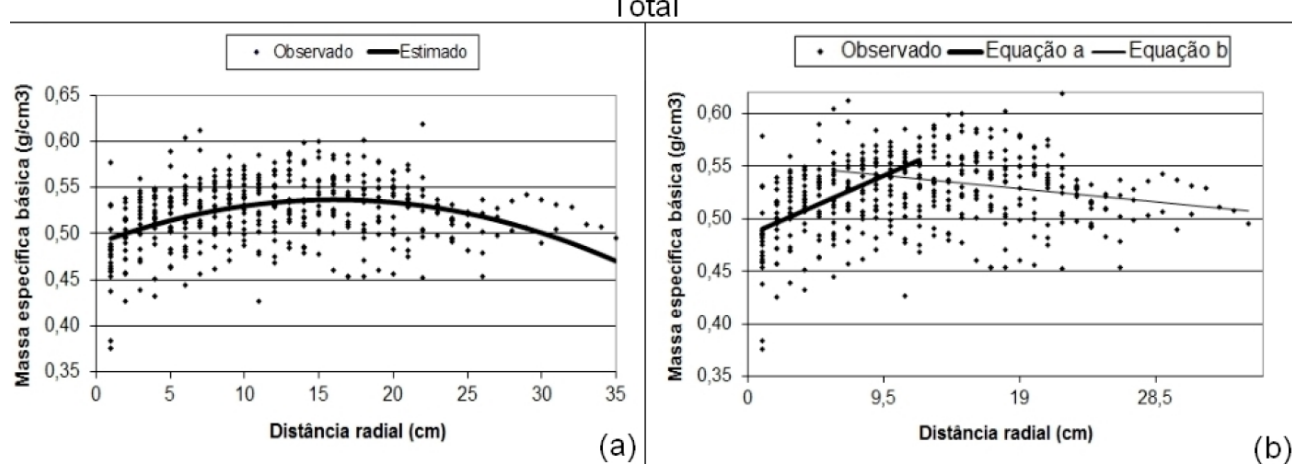

Figura 3 - Variação radial da massa específica nas duas Regiões e no total de árvores, sendo índices (a) e (b) os dados observados e a estimativa de segregação do lenho, respectivamente.

Ciência Rural, v.42, n.9, set, 2012. 
Regiões, bem como para o conjunto de árvores analisadas. São apresentados os dados observados no estudo nos gráficos com índices (a) e os modelos de regressão linear estimando a idade de segregação do lenho nos gráficos de índices (b). As equações das retas de regressão são apresentadas na tabela 3 .

Pode-se observar que, para a região da

Depressão Central, a madeira de açoita-cavalo

Tabela 3 - Modelos de regressão individuais, por região, e total para a estimação da idade e posição de segregação da madeira de açoita-cavalo.

\begin{tabular}{|c|c|c|c|c|c|c|c|c|}
\hline Árvore & $\begin{array}{l}\text { Segregação } \\
(\mathrm{mm})\end{array}$ & $\begin{array}{l}\text { Segregação } \\
\text { (anel) }\end{array}$ & & Equação & $\mathrm{R}^{2} \mathrm{aj}(\%)$ & Sxy & $\mathrm{F}$ & $\mathrm{P}$ \\
\hline \multirow{2}{*}{1} & \multirow{2}{*}{130} & \multirow{2}{*}{35} & $\mathrm{a}$ & $\mathrm{ME}=0,463077+0,00706593 *$ posição & 59 & 0,02 & 40 & 0,00 \\
\hline & & & $\mathrm{b}$ & $\mathrm{ME}=0,58928-0,00210862 *$ posição & 46 & 0,02 & 18 & 0,00 \\
\hline \multirow{2}{*}{2} & \multirow{2}{*}{60} & \multirow{2}{*}{19} & $\mathrm{a}$ & $\mathrm{ME}=0,495694+0,00575 *$ posição & 37 & 0,02 & 11 & 0,00 \\
\hline & & & $\mathrm{b}$ & $\mathrm{ME}=0,529895$ & - & 0,09 & - & 0,00 \\
\hline \multirow{2}{*}{3} & \multirow{2}{*}{100} & \multirow{2}{*}{26} & a & $\mathrm{ME}=0,4889$ & - & 0,01 & - & 0,00 \\
\hline & & & $\mathrm{b}$ & $\mathrm{ME}=0,522492-0,00343849 *$ posição & 51 & 0,01 & 15 & 0,00 \\
\hline \multirow{2}{*}{4} & \multirow{2}{*}{100} & \multirow{2}{*}{26} & a & $\mathrm{ME}=0,474722+0,00716667 *$ posição & 32 & 0,03 & 9 & 0,01 \\
\hline & & & $\mathrm{b}$ & $\mathrm{ME}=0,585663-0,00378254 *$ posição & 24 & 0,03 & 10 & 0,00 \\
\hline \multirow{2}{*}{5} & \multirow{2}{*}{70} & \multirow{2}{*}{22} & a & $\mathrm{ME}=0,501111+0,004^{*}$ posição & 29 & 0,02 & 8 & 0,01 \\
\hline & & & $\mathrm{b}$ & $\mathrm{ME}=0,528776$ & - & 0,02 & - & 0,00 \\
\hline \multirow{2}{*}{6} & \multirow{2}{*}{120} & \multirow{2}{*}{28} & a & $\mathrm{ME}=0,485278+0,0075^{*}$ posição & 19 & 0,04 & 5 & 0,04 \\
\hline & & & $\mathrm{b}$ & $\mathrm{ME}=0,608045-0,00278961 *$ posição & 23 & 0,02 & 7 & 0,02 \\
\hline \multirow{2}{*}{7} & \multirow{2}{*}{60} & \multirow{2}{*}{18} & a & $\mathrm{ME}=0,428889+0,0193333 *$ posição & 56 & 0,04 & 23 & 0,00 \\
\hline & & & $\mathrm{b}$ & $\mathrm{ME}=0,551163-0,00173573 *$ posição & 2 & 0,02 & 11 & 0,00 \\
\hline \multirow{2}{*}{8} & \multirow{2}{*}{135} & \multirow{2}{*}{34} & a & $\mathrm{ME}=0,517527+0,0029011 *$ posição & 16 & 0,03 & 6 & 0,02 \\
\hline & & & $\mathrm{b}$ & $\mathrm{ME}=0,604447-0,00348136 *$ posição & 18 & 0,03 & 7 & 0,01 \\
\hline \multirow{2}{*}{9} & \multirow{2}{*}{130} & \multirow{2}{*}{26} & a & $\mathrm{ME}=0,505875+0,00151471^{*}$ posição & 8 & 0,03 & 5 & 0,02 \\
\hline & & & $\mathrm{b}$ & $\mathrm{ME}=0,526473$ & - & 0,01 & - & 0,00 \\
\hline \multirow{2}{*}{10} & \multirow{2}{*}{90} & 29 & a & $\mathrm{ME}=0,465+0,00933333^{*}$ posição & 57 & 0,02 & 23 & 0,00 \\
\hline & & 29 & $\mathrm{~b}$ & $\mathrm{ME}=0,5532$ & - & 0,02 & - & 0,00 \\
\hline Média & 99,5 & 26,3 & & & & & & \\
\hline Regiões & $\begin{array}{l}\text { Segregação } \\
\quad(\mathrm{mm})\end{array}$ & $\begin{array}{l}\text { Segregação } \\
\quad \text { (anel) }\end{array}$ & & Equação & $\mathrm{R}^{2} \mathrm{aj}(\%)$ & Sxy & $\mathrm{F}$ & $\mathrm{P}$ \\
\hline Den Central & 90 & 26 & a & $\mathrm{ME}=0,483472+0,00475 *$ posição & 14 & 0,03 & 16 & 0,00 \\
\hline Dep. central & 90 & 26 & $\mathrm{~b}$ & $\mathrm{ME}=0,5265$ & - & 0,01 & - & 0,00 \\
\hline & Estimado & & & $\begin{array}{l}\text { ME }=0,481423+0,00619995 * \text { posição - } \\
0,00020253^{*} \text { posição^2 }\end{array}$ & 12 & 0,03 & 14 & 0,00 \\
\hline Enc. Sup. & 95 & 23 & a & $\mathrm{ME}=0,484778+0,0072 *$ posição & 20 & 0,04 & 23 & 0,00 \\
\hline Nordeste & 95 & 23 & $\mathrm{~b}$ & $\mathrm{ME}=0,572704-0,00201695^{*}$ posição & 15 & 0,03 & 24 & 0,00 \\
\hline & & & Estimado & $\begin{array}{l}\mathrm{ME}=0,494804+0,00631103 * \text { posição }- \\
0,00019958 * \text { posição^} 2^{\wedge}\end{array}$ & 18 & 0,03 & 25 & 0,00 \\
\hline & & & a & $\mathrm{ME}=0,484125+0,005975 *$ posição & 17 & 0,03 & 37 & 0,00 \\
\hline Total & 95 & 26 & $\mathrm{~b}$ & $\mathrm{ME}=0,55361-0,00132293^{*}$ posição & 4 & 0,03 & 10 & 0,00 \\
\hline & & & Estimado & $\begin{array}{l}\mathrm{ME}=0,489074+0,00596994 * \text { posição - } \\
0,000185993 * \text { posição }^{\wedge} 2\end{array}$ & 14 & 0,03 & 35 & 0,00 \\
\hline
\end{tabular}

Em que: $\mathrm{R}_{\mathrm{aj}}^{2}=\mathrm{R}$ quadrado ajustado (\%); $\mathrm{S}_{\mathrm{xy}}=$ Erro padrão de estimativa; F=Valor F calculado; $\mathrm{P}=$ =Probabilidade.

Ciência Rural, v.42, n.9, set, 2012. 
segregou a $9 \mathrm{~cm}$ da medula, com 26 anos. Por outro lado, nota-se que, para a região da Encosta Superior do Nordeste, houve a separação entre lenho juvenil e adulto na posição de 9,5cm, a partir da medula, aos 23 anos. Para o conjunto de árvores analisadas, tem-se um resultado semelhante, sendo 26 anos a idade de segregação dos lenhos, a 9,5cm da medula.

A média aritmética do ano de segregação do total de árvores, correspondente a 26,3 anos, é semelhante ao valor encontrado por regressão linear, para a região da Depressão Central, de 26 anos. Entretanto, difere do valor de 23 anos apresentado na região da Encosta Superior do Nordeste.

Segundo DINWOODIE (1981), as alterações no volume de espaços vazios no interior da madeira e na espessura da parede celular ocasionam variações de massa específica. Tais alterações são proporcionadas por tratos silviculturais, idade da árvore, condições ambientais e fatores genéticos. As diferentes combinações desses parâmetros justificam as diferenças estatísticas entre as massas específicas das duas Regiões, além de explicar a variação na idade de segregação entre os dois grupos analisados neste estudo.

A determinação da idade de segregação do lenho de açoita-cavalo foi determinada por GATTO et al. (2008) através da análise de parâmetros anatômicos, sendo observada uma idade de segregação de 21 anos. No presente trabalho, obteve-se como valor médio uma idade de segregação de 26,3 anos, diferença justificada pela divergência de metodologias.

\section{CONCLUSÃO}

As árvores da região da Depressão Central apresentaram idade de segregação de 26 anos, sendo esse valor superior ao dado encontrado para as árvores da região da Encosta Superior do Nordeste, cuja idade corresponde a 23 anos.

Os dados médios de massa específica para as duas Regiões e para os dois tipos de lenho apresentam diferença estatística entre si. Entretanto, não há diferença estatisticamente significativa entre os dados médios de massa específica para as amostras A e B.

\section{REFERÊNCIAS}

ABDEL-GADIR, A.Y.; KRAHMER, R.L. Estimating the age of demarcation of juvenile and mature wood in Douglas-fir. Wood and Fiber Science, v.25, n.3, p.242-249, 1993. Disponível em: <https://swst.metapress.com/content/q02j065x61715342/ resource-secured/?target=fulltext.pdf $>$. Acesso em: 4 jun. 2012.

ALZATE, S.B.A. et al. Variação longitudinal da densidade básica da madeira de clones de Eucalyptus grandis Hill ex Maiden, $\boldsymbol{E}$. saligna Sm. e E. grandis x urophylla. Scientia Forestalis, n.68, p.87-95, 2005. Disponível em: <http://www.ipef.br/ publicacoes/scientia/nr68/cap08.pdf>. Acesso em: 4 jun. 2012.

ASTM (American Society for Testing and Materials). Specific gravity of wood and wood-based materials: ASTM D2395 - 93. Philadelphia, PA, 1997. 8p.

Sampling forest trees for determination of clear wood properties: ASTM D5536-94. Philadelphia, PA, 1999. 9p.

BENDTSEN, B.A.; SENFT, J. Mechanical and anatomical properties in individual growth rings of plantation-grown cottonwood and loblolly pine. Wood Fiber Science, v.18, n.1, p.23-28, 1986. Disponível em: <https:// swst.metapress.com/content/mqk614333w4k5q65/resourcesecured/?target=fulltext.pdf $>$. Acesso em: 4 jun. 2012.

COPANT (Comisión Panamericana de Normas Técnicas). Selección y colección de maderas: COPANT 30:1- 001. [S.l.], 1971.

DINWOODIE, J.N. Timber its nature and behavior. New York: Reinhold, 1981. 190p.

GATTO, D.A. et al. Estimativa da idade de segregação do lenho juvenil a adulto por meio de parâmetros anatômicos para madeira de Luehea divaricata Mart. Ciência Florestal, v.18, n.4, p.535-540, 2008. Disponível em: <http://cascavel.ufsm.br/ revistas/ojs-2.2.2/index.php/cienciaflorestal/article/view/436/ 321>. Acesso em: 4 jun. 2012.

GATTO, D.A. et al. Características tecnológicas das madeiras de Luehea divaricata, Carya illinoinensise e Platanus $x$ acerifolia quando submetidas ao vergamento. Ciência Florestal, v.18, n.1, p.121-131, 2008. Disponível em: <http:/ /www.ufsm.br/cienciaflorestal/artigos/v18n1/A11V18N1.pdf>. Acesso em: 4 jun. 2012.

KOLLMANN, F.F.P.; CÔTÉ JUNIOR, W.A. Principles of wood science and technology. Berlin: Springer-Verlag, 1968. 703p.

OLIVEIRA, J.T.S.; SILVA, J.C. Variação radial da retratibilidade e densidade básica da madeira de Eucalyptus saligna Sm. Revista Árvore, v.27, n.3, p.381-385, 2003. Disponível em: <http://www.scielo.br/ pdf/rarv/v27n3/a15v27n3.pdf>. Acesso em: 4 jun. 2012.

PANSHIN, A.J.; DE ZEEUW, C. Text book of wood technology. 3.ed. New York: Mc-Graw-Hill, 1970. 705p.

RAMSAY, W.; BRIGGS, D. Juvenile wood: has it come of age. In: A TECHNICAL WORKSHOP: JUVENILE WOOD-WHAT DOES IT MEAN TO FOREST MANAGEMENT AND FOREST PRODUCTS, 1985, Washington. Proceedings... Madison: Forest Products Research Society, 1986. p.5-11.

SILVA, J.C. et al. Influência da idade e da posição radial na massa específica da madeira de Eucalyptus grandis Hill ex. MAIDEN. Revista Floresta, v.34, n.1, p.13-22, 2004. Disponível em: <http://ojs.c3sl.ufpr.br/ojs2/index.php/floresta/ article/view/2371/1980>. Acesso em: 4 jun. 2012.

VALE, A.T. et al. Massa específica básica da madeira de Pinus caribaea var. hondurensis cultivado em cerrado. Scientia Forestalis, v.37, n.84, p.387-394, 2009. Disponível em: <http:/ /www.ipef.br/publicacoes/scientia/nr84/cap06.pdf>. Acesso em: 4 jun. 2012. 